\title{
Biexcitons or bipolaritons in a semiconductor microcavity
}

Borri, Paola; Langbein, Wolfgang Werner; Woggon, U; Jensen, Jacob Riis; Hvam, Jørn Märcher

Published in:

Physical Review B Condensed Matter

Link to article, DOI:

10.1103/PhysRevB.62.R7763

Publication date:

2000

Document Version

Publisher's PDF, also known as Version of record

Link back to DTU Orbit

Citation (APA):

Borri, P., Langbein, W. W., Woggon, U., Jensen, J. R., \& Hvam, J. M. (2000). Biexcitons or bipolaritons in a semiconductor microcavity. Physical Review B Condensed Matter, 62(12), R7763-R7766.

https://doi.org/10.1103/PhysRevB.62.R7763

\section{General rights}

Copyright and moral rights for the publications made accessible in the public portal are retained by the authors and/or other copyright owners and it is a condition of accessing publications that users recognise and abide by the legal requirements associated with these rights.

- Users may download and print one copy of any publication from the public portal for the purpose of private study or research.

- You may not further distribute the material or use it for any profit-making activity or commercial gain

- You may freely distribute the URL identifying the publication in the public portal 


\title{
Biexcitons or bipolaritons in a semiconductor microcavity
}

\author{
P. Borri, W. Langbein, and U. Woggon \\ Experimentelle Physik EIIb, Universität Dortmund, Otto-Hahn Strasse 4, 44227 Dortmund, Germany \\ J. R. Jensen and J. M. Hvam \\ Research Center COM, The Technical University of Denmark, Building 349, DK-2800 Kgs. Lyngby, Denmark
}

(Received 19 July 2000)

\begin{abstract}
A well-resolved nonlinear optical transition associated with biexcitons is observed in a high-quality microcavity with a Rabi splitting exceeding the binding energy of biexcitons in the embedded quantum well. This transition is identified as an induced absorption from the lower polariton to the biexciton. The biexciton binding energy is found not to be significantly affected by the coupling with the cavity photons. In spite of the high quality of the sample, formation of a bipolariton as a bound state of two lower cavity polaritons with small in-plane wave vectors is not resolved.
\end{abstract}

The formation of biexcitons in semiconductors has attracted considerable attention in recent years. ${ }^{1}$ Especially intriguing is, from the fundamental point of view, the problem of a biexciton in the presence of two types of interactions: the exciton-exciton attraction by the Coulomb interaction between the constituent electrons and holes, leading to the biexciton formation, and the exciton-photon coupling which leads to the formation of polaritons. ${ }^{2}$ The possibility to realize low-dimensional heterostructures has introduced a further variable to the problem. In low-dimensional systems, such as quantum wells (QW's), the Coulomb interaction is enhanced by the spatial confinement. The interaction of excitons with light is also strongly modified by the confinement. In twodimensional (2D) systems, the breaking of translational invariance along the growth direction leads to the coupling to a continuum of photon modes, resulting in an irreversible radiative decay of the excitons, while in the bulk case the polariton is a stationary state. ${ }^{2}$ The influence of the excitonphoton coupling on the internal structure of biexcitons has been discussed in 3D, 2D, and $1 \mathrm{D}$ systems. ${ }^{3}$

The recent development of microcavity structures has opened new possibilities to control the light-matter interaction. ${ }^{4,5}$ A quantum-well microcavity is a planar FabryPerot cavity with an optical length of an integer multiple of half-wavelengths of the photons resonant to the QW excitons embedded in the cavity. Photon quantization along the cavity axis occurs, and excitons with a given in-plane wave vector couple to only one photon mode. Stationary eigenstates of the system now exist, called cavity polaritons, and an irreversible radiative decay of excitons no longer occurs. In real systems the photons in the cavity have a finite lifetime due to transmission through the mirrors. Thus, cavity polaritons are converted into external photons as a result of the finite lifetime of their photonic component in the cavity. The strong exciton-photon coupling that occurs in a microcavity significantly modifies the optical properties of the embedded QW excitons. When the vacuum Rabi splitting in the microcavity, which quantifies the strength of the exciton-photon coupling, is significantly bigger than the biexciton binding energy one would imagine that the optical properties of biexcitons are also affected by the coupling with the cavity photons. In particular, the formation of a bound two-polariton system (bipolariton) could be expected.

The experimental observation of biexcitons in microcavity was addressed recently by Fan $e t a .^{6}$ and by Neukirch et $a .^{7}$ Fan et al. could not distinguish a well-resolved biexciton transition due to a large broadening of the investigated polariton resonances. Alternatively, Neukirch et al. used a nonmonolithic II-VI microcavity with a biexciton binding energy that exceeds the vacuum Rabi splitting and all damping constants. This structure allowed them to spectrally separate biexcitonic contributions from the polariton resonances. However, such a structure is not suitable to investigate the effect of a strong exciton-photon coupling on the binding of biexcitons in microcavities, which was, thus, not addressed in their work. They measured coherent spectral oscillations in a pump-probe experiment at negative pump-probe delay times which included coherent features related to an upper polariton-biexciton transition. However, an incoherent pump-induced absorption associated with this transition at positive delay times was not observed.

In this paper we investigate the nonlinear optical properties of a very high-quality monolithic QW microcavity in a pump-probe experiment. The heavy-hole vacuum Rabi splitting in the microcavity is approximately four times bigger than the biexciton binding energy in the bare QW. Due to the narrow linewidth of the polariton resonances, a well-resolved pump-induced optical absorption associated with biexcitons is observed. The energy position, the dependence on the polarization of pump and probe photons, and the dependence on the detuning between the cavity mode and the exciton clearly demonstrate the biexcitonic nature of this resonance. The biexciton binding energy is found not to be significantly affected by the strong exciton-photon coupling in the microcavity.

The sample consists of a molecular-beam epitaxy-grown $25 \mathrm{~nm} \mathrm{GaAs} / \mathrm{Al}_{0.3} \mathrm{Ga}_{0.7} \mathrm{As}$ single quantum well placed in the center of a $\lambda$ cavity. ${ }^{8}$ An $\mathrm{AlAs} / \mathrm{Al}_{0.15} \mathrm{Ga}_{0.85} \mathrm{As}$ Bragg reflector of 25 (16) periods was grown at the bottom (top) of the cavity. The $\mathrm{Al}_{0.3} \mathrm{Ga}_{0.7} \mathrm{As}$ barriers of the $\mathrm{QW}$ are $5 \mathrm{~nm}$ thick and the rest of the spacer layer consists of $\mathrm{Al}_{0.05} \mathrm{Ga}_{0.95} \mathrm{As}$. The spacer layer is wedged, in order to tune the cavity mode 


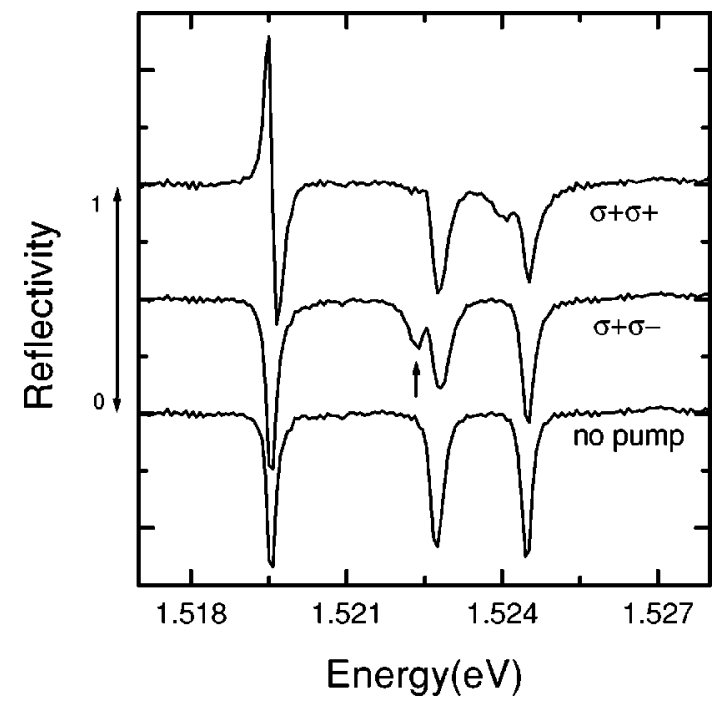

FIG. 1. Reflectivity spectra for co-circular and cross-circular pump and probe photons at zero pump-probe delay. The cavity mode is in resonance with the bare $\mathrm{HH}$ exciton (zero detuning). The pump fluence is $0.12 \mu \mathrm{J} / \mathrm{cm}^{2}$. The lower curve is the reflectivity spectrum without the pump pulse, shown for reference.

along the position on the sample. Details on the growth conditions and sample design can be found in Ref. 9. The pumpprobe experiment has been performed using a Ti:sapphire laser source providing Fourier-limited 100 fs laser pulses at $76 \mathrm{MHz}$ repetition rate. The spectrum of the pump pulse was shaped in order to resonantly excite only the lower polariton in the microcavity, resulting in a pulse duration of $\sim 500 \mathrm{fs}$. Small incidence angles $\left(\sim 1^{\circ}\right)$ for both pump and probe were used and the probe reflectivity was spectrally analyzed with a spectrometer and a charge-coupled device camera of $70 \mu \mathrm{eV}$ resolution (half width at half maximum-HWHM). The probe spot size was $\sim 40 \mu \mathrm{m}$, resulting in a small energy broadening of the cavity mode from the wedge gradient and the incident wave-vector spreading. ${ }^{9}$ The pump spot size was chosen $40 \%$ bigger than the probe, in order to have a uniform pump density over the probe area. The sample was held in a helium bath cryostat at $T=5 \mathrm{~K}$ for all the measurements. A variable delay time between pump and probe pulses and Babinet-Soleil plates for polarization control were used.

The probe reflectivity spectrum without the pump pulse is shown in Fig. 1 with the cavity mode in resonance with the bare heavy-hole (HH) quantum-well exciton (zero detuning). The wide GaAs quantum well has a narrow and homogeneously broadened bare QW exciton absorption resonance. ${ }^{10}$ The strong exciton-photon coupling in the microcavity structure leads to three polariton resonances, arising from the mixing of the heavy-hole exciton, the light-hole (LH) exciton and the cavity mode. Measurements at $11 \mathrm{~K}$ of the polariton resonances for different detuning between the cavity mode and the $\mathrm{HH}$ exciton were fitted by a three coupled-oscillator model from which we have inferred a Rabi splitting of 3.6 $\mathrm{meV}$ for the $\mathrm{HH}$ and $2.2 \mathrm{meV}$ for the LH excitons. ${ }^{9}$ Moreover, the use of a high resolution spectrometer allowed us to accurately measure the polariton linewidth, which was found to be $95 \mu \mathrm{eV}$ HWHM at each of the resonances at zero detuning, resulting in a ratio of 19 between the HH Rabi

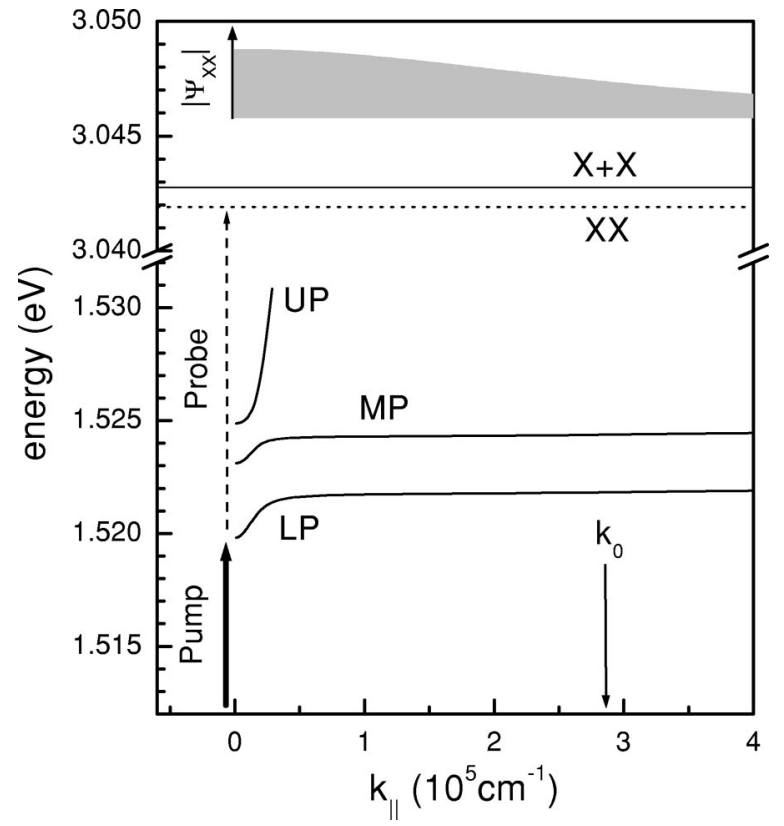

FIG. 2. Lower (LP), middle (MP), and upper polariton (UP) dispersion versus the in-plane wave vector. The radiative cutoff $k_{0}$ is indicated. The biexciton $(X X)$ and twice the exciton energies $(X+X)$ are also shown. The amplitude of the biexciton wave function of the exciton-exciton relative motion extending to large wave vectors of one of the constituent excitons, comparable to the radiative cutoff, is depicted in gray scale. The absorption of pump photons (solid arrow) creates a LP density that induces a LP- $X X$ absorption of probe photons (dashed arrow).

splitting and the linewidth. ${ }^{11}$ The probe reflectivity in presence of the pump pulse at zero relative delay is shown in Fig. 1 for two circular polarization combinations of pump and probe as indicated. The pump pulse leads to a well distinguished absorption resonance slightly below the middle polariton (MP) that disappears for co-circular polarizations (see arrow in figure). ${ }^{12}$ The formation of heavy-hole biexcitons in a bare QW is known to follow these polarization selection rules, namely, is canceled for co-circular excitation. ${ }^{10}$

In a recent theoretical work, ${ }^{13}$ the main difference between the lowest bipolariton bound state in a microcavity, formed by two lower polaritons (LP) with small in-plane wave vectors $\left(\mathbf{k}_{\|}\right)$, and the bare biexciton has been pointed out. The total energy of the bipolariton is twice the LP transition energy minus the bipolariton binding. Thus, by populating the LP branch at small $\mathbf{k}_{\|}$with the pump pulse, an induced absorption from the LP to the bipolariton should lead to a reflectivity dip below the LP, separated by the bipolariton binding. However, due to the small effective mass in the LP, the bipolariton binding energy is very small compared to the bare biexciton value. It scales exponentially with the mass ratio between LP and exciton and is negligible compared to the unavoidable damping of the LP, ${ }^{13}$ even at very low temperatures due to the radiative damping. Thus, a bipolariton resonance is not expected to be resolved in microcavities. Instead, the bare biexciton radius, typically comparable to the exciton Bohr radius, corresponds to a biexciton wave function that extends to large in-plane wave vectors of the constituent excitons $\left(\mathbf{k}_{\|} \sim 2 \pi n / \lambda\right)$. Therefore, even inside the microcavity, the biexciton bound-state structure 


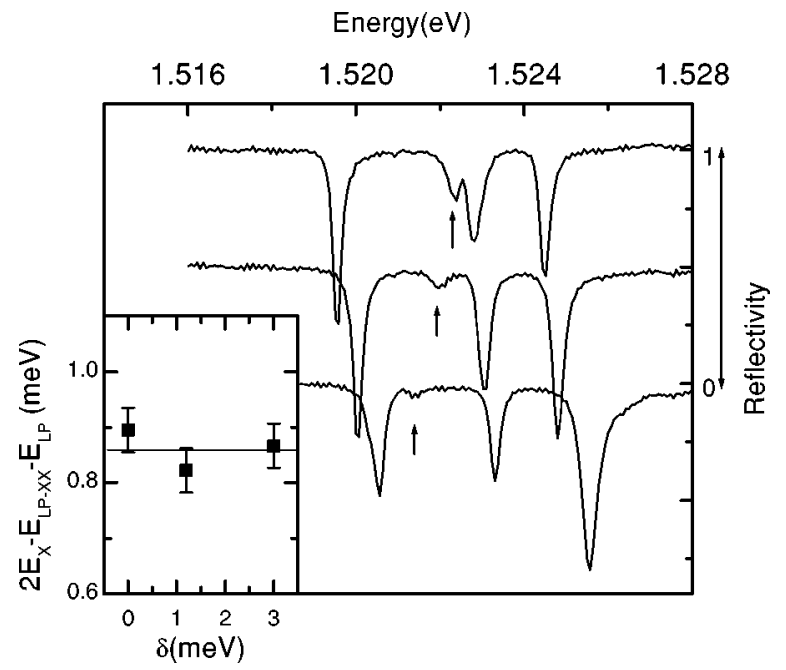

FIG. 3. Reflectivity spectra at zero pump-probe delay for different detuning between the cavity mode and the $\mathrm{HH}$ exciton. The pump fluence is $0.12 \mu \mathrm{J} / \mathrm{cm}^{2}$. The inset shows the difference between twice the $\mathrm{HH}$ bare exciton energy and the measured sum of the LP and LP- $X X$ transition energies. The line is a guide for the eyes. The detuning $\delta$ is defined as the energy difference between the cavity mode and the bare $\mathrm{HH}$ exciton.

(i.e., binding energy and radius) is not expected to be significantly modified by the photon coupling which is affecting only the dispersion region at much smaller $\mathbf{k}_{\|} \cdot{ }^{13}$ The transition from the LP to the biexciton is higher in energy than the LP transition, ${ }^{14}$ by the amount: $E_{\mathrm{LP}-X X}-E_{\mathrm{LP}}=2\left(E_{X}-E_{\mathrm{LP}}\right)$ $-E_{B X X}$, with $E_{\mathrm{LP}}, E_{X}, E_{\mathrm{LP}-X X}$ being the lower polariton, bare exciton, and LP-biexciton transition energies, respectively, and $E_{B X X}$ the biexciton binding energy. These concepts are sketched in Fig. 2. The dispersion of the LP, MP, and upper polariton (UP) branches are calculated for the investigated structure at zero detuning. The radiative cutoff of the exciton-photon coupling $k_{0}=2 \pi n / \lambda$ is indicated. The biexciton energy $(X X)$ and twice the bare exciton energy $(X+X)$ are indicated, the difference between them being the biexciton binding energy. The pump photons create a LP density at $\mathbf{k}_{\|} \sim 0$. When the probe arrives, an induced absorption from the LP to the biexciton transition occurs. The amplitude of the optically active biexciton wave function with the wave vector $K \sim 0$ of the in-plane center of mass motion is also shown versus the in-plane wave vector of one of the constituent excitons. This wave function describes the exciton-exciton relative motion with a relative wave vector, at $K=0$, which is twice the one of the constituent exciton. We have calculated this wave function using the $2 \mathrm{D}$ deuterium ground-state formula with the biexciton radius given by $1 / \sqrt{8} k_{0}$ that follows from equal radiative rates of the biexciton and the exciton, ${ }^{1}$ as experimentally measured in a bare QW. ${ }^{10}$ The biexciton wave function extends to large wave vectors, even above the radiative cutoff.

That the observed induced absorption shown in Fig. 1 corresponds to the LP- $X X$ transition is further confirmed by changing the detuning between the cavity mode and the $\mathrm{HH}$ exciton. When shifting the cavity mode above the HH exciton (positive detuning) the LP transition shifts towards higher energies. ${ }^{9}$ Since the $X X$ energy is not significantly affected by the coupling to the cavity photons and therefore
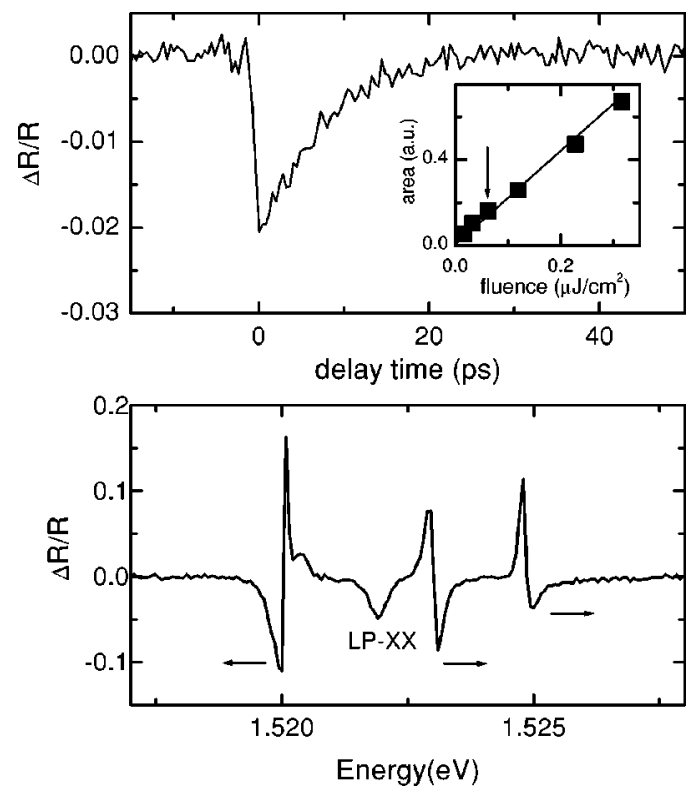

FIG. 4. Upper plot: Differential reflectivity time-resolved and spectrally integrated over the LP- $X X$ transition. Inset: timeintegrated differential reflectivity versus pump fluence. The arrow indicates the fluence used in the curve shown. Lower plot: Differential reflectivity spectrum at zero delay. The arrows indicate the shifts of the LP, MP, and UP that occur in presence of the pump pulse. All data are taken with the cavity mode $1.2 \mathrm{meV}$ above the bare $\mathrm{HH}$ exciton (positive detuning).

by the detuning, the LP- $X X$ transition should shift towards lower energies. This is shown in Fig. 3 for three different detunings. The LP- $X X$ transition is indicated by the arrows, and clearly shifts towards lower energies while the LP shifts towards higher energies. This also results in a reduced visibility of the LP- $X X$ resonance while it shifts far from the cavity resonance. The expected shift of the LP- $X X$ transition versus the detuning is given by $E_{\mathrm{LP}-X X}=2 E_{X}-E_{B X X}-E_{\mathrm{LP}}$ with the detuning dependent $E_{\mathrm{LP}}$. Thus, the sum of $E_{\mathrm{LP}-X X}$ and $E_{\mathrm{LP}}$ is expected to be independent of the detuning. In the inset of Fig. 3 the difference between twice the bare $\mathrm{HH}$ exciton energy and the measured sum of LP and LP- $X X$ transition energies is shown versus the detuning. Within the experimental uncertainty, a constant biexciton binding energy of $0.86 \mathrm{meV}$ is obtained. This value is slightly smaller than the $1.1 \mathrm{meV}$ measured in a comparable bare QW. ${ }^{10} \mathrm{We}$ attribute the reduction to an increased dielectric screening of the Coulomb attraction, as a consequence of the higher refractive index of the QW barrier in the microcavity structure compared to the bare QW. ${ }^{9,10}$ Additionally, built-in electric fields would also reduce the biexciton binding. The presence of excess free carriers, that could lead to such electric fields, was in fact observed in the investigated structure. ${ }^{11} \mathrm{~A}$ constant value of $E_{B X X}$, independent of the detuning, shows the negligible influence of the exciton-photon coupling to the biexciton binding.

The dynamics of the LP- $X X$ induced absorption is ruled by the LP lifetime. When the LP density created by the pump is fully decayed into external photons via the cavity photon lifetime, the LP- $X X$ induced transition disappears. This is, in fact, observed by measuring the induced absorption as a function of the pump-probe delay (see Fig. 4). Positive delay 
corresponds to the pump leading the probe pulse. In the top part of Fig. 4 is shown the differential reflectivity at the LP$X X$ transition, spectrally integrated over the resonance. The lifetime of the induced absorption is well fitted by an exponential decay with a time constant of $6.5 \pm 0.5 \mathrm{ps}$. The cavity photon lifetime in the investigated structure is $2.2 \mathrm{ps}$, as deduced by the cavity linewidth of Ref. $9 \sim 300 \mu \mathrm{eV}$. At the detuning corresponding to the data shown in Fig. 4 we calculated a photonic content of 0.37 in the LP that results in

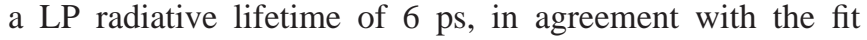
within errors. In the inset is shown the dependence of the time-integrated differential reflectivity at the LP- $X X$ transition on the pump excitation intensity. A linear dependence is observed, as expected from a LP- $X X$ absorption proportional to the pump-induced LP density.

Finally, the lower part of Fig. 4 shows the spectrally resolved differential reflectivity at zero delay. Compared to the direct reflectivity data, the LP- $X X$ transition is resolved with better signal-to-noise ratio in the differential reflectivity spectrum. The LP- $X X$ energy positions used in the inset of Fig. 3 were measured from the differential reflectivity spectra to reduce the uncertainty. An additional feature is observed in the spectrum. In the presence of the pump pulse the LP, MP, and UP energies are slightly shifted (shifts are below $100 \mu \mathrm{eV}$ ) as indicated by the arrows, corresponding to an increase of the $\mathrm{HH}$ rabi splitting. This was observed at all investigated pump intensities at $1.2 \mathrm{meV}$ and $3 \mathrm{meV}$ detunings, but less pronounced at zero detuning. Similar observations have been reported, ${ }^{6}$ which were, however, based on experimental findings dominated by the inhomogeneous broadening in the microcavity resonances, and attributed to the induced biexciton transition within a phenomenological model. Based on the high quality of the presented data, further theoretical work for a detailed understanding of this effect is strongly encouraged.

In conclusion, we have experimentally shown the presence of a well-resolved nonlinear optical transition associated with biexcitons in a quantum-well microcavity of high quality in the strong coupling regime. This transition is identified as an induced absorption from the lower polariton to the biexciton. Even if the vacuum Rabi splitting in the microcavity is four times bigger than the biexciton binding energy in the bare quantum well, the biexciton binding energy is not significantly affected by the coupling with the cavity photons. In contrast, the formation of a bipolariton as a bound state of two lower cavity polaritons with small inplane wave vectors is not resolved. These results are in agreement with theoretical expectations in literature based on the in-plane wave-vector dispersion in microcavities. ${ }^{13,14}$
${ }^{1}$ A. L. Ivanov, H. Haug, and L. V. Keldysh, Phys. Rep. 296, 237 (1998).

${ }^{2}$ L. C. Andreani, in Confined Electrons and Photons: New Physics and Applications, Vol. 340 of NATO Science Series B: Physics, edited by E. Burstein and C. Weisbuch (Plenum Press, New York, 1995), pp. 57-112.

${ }^{3}$ A. L. Ivanov and H. Haug, Phys. Rev. Lett. 74, 438 (1995).

${ }^{4}$ M. S. Skolnick, T. A. Fisher, and D. M. Whittaker, Semicond. Sci. Technol. 13, 645 (1998).

${ }^{5}$ G. Khitrova, H. M. Gibbs, F. Jahnke, M. Kira, and S. W. Koch, Rev. Mod. Phys. 71, 1591 (1999).

${ }^{6}$ X. Fan, H. Wang, H. Q. Hou, and B. E. Hammons, Phys. Rev. B 57, R9451 (1998).

${ }^{7}$ U. Neukirch, S. R. Bolton, N. A. Fromer, L. J. Sham, and D. S. Chemla, Phys. Rev. Lett. 84, 2215 (2000).

${ }^{8}$ The sample was grown at III-V Nanolab run jointly by Research Center COM and the Niels Bohr Institute at Copenhagen University.
${ }^{9}$ J. R. Jensen, P. Borri, W. Langbein, and J. M. Hvam, Appl. Phys. Lett. 76, 3262 (2000).

${ }^{10}$ W. Langbein and J. M. Hvam, Phys. Rev. B 61, 1692 (2000).

${ }^{11}$ P. Borri, J. R. Jensen, W. Langbein, and J. M. Hvam, Phys. Rev. B 61, R13 377 (2000).

${ }^{12}$ For co-circular polarizations an induced absorption slightly below the upper polariton is also observed. We suggest that it corresponds to a heavy-hole light-hole mixed biexciton, which is allowed for co-circular polarizations and is separated from the $\mathrm{HH}$ biexciton by the HH-LH splitting. Moreover, a strong contribution from coherent nonlinearities is observed at the LP; that follows the selection rules of a four-wave mixing signal, namely is suppressed for cross-circular polarizations.

${ }^{13}$ G. C. L. Rocca, F. Bassani, and V. M. Agranovich, J. Opt. Soc. Am. B 15, 652 (1998).

${ }^{14}$ C. Sieh, T. Meier, A. Knorr, F. Jahnke, P. Thomas, and S. W. Koch, Eur. Phys. J. B 11, 407 (1999). 